\title{
Intracardiac thrombus in a patient with catastrophic antiphospholipid syndrome: an autopsy case report and review of the literature
}

This article was published in the following Dove Press journal:

Vascular Health and Risk Management

\author{
Jariya Waisayarat $\mathbb{D}^{\prime}$ \\ Sirithep Plumworasawat' \\ Soamarat Vilaiyuk (iD ${ }^{2}$ \\ Nongnuch Sirachainan $\mathbb{D}^{2}$ \\ 'Department of Pathology, Faculty of \\ Medicine Ramathibodi Hospital, Mahidol \\ University, Bangkok, Thailand; \\ ${ }^{2}$ Department of Pediatrics, Faculty of \\ Medicine Ramathibodi Hospital, Mahidol \\ University, Bangkok, Thailand
}

\begin{abstract}
Antiphospholipid syndrome (APS) is an autoantibody-mediated acquired thrombophilia characterized by venous and/or arterial thromboses, pregnancy morbidity (predominantly repeated fetal losses), and the presence of phospholipid antibodies. The estimated annual incidence of APS is 5 new cases per 100,000 people. The most common thrombotic events in patients with APS in order of frequency are stroke, transient ischemic attack, deep vein thrombosis, and pulmonary embolism. Patients with APS may develop an intracardiac thrombus, which is a life-threatening complication with a high risk of increased morbidity and mortality; however, it is treatable by surgical removal, extensive anticoagulant administration, and prevention of other complications. Catastrophic APS, which is a rare and severe condition diagnosed based on rapidly progressive thromboembolic events involving three or more organs, systems, or tissues, occurs in less than $1 \%$ of all patients with APS. We herein report an autopsy case of catastrophic APS in a 12-year-old Thai boy with multiple thromboembolic events including intracardiac thrombus formation with a positive lupus anticoagulant test result. To the best of our knowledge, this is the youngest reported patient with APS to date.
\end{abstract}

Keywords: antiphospholipid syndrome, intracardiac thrombus, catastrophic antiphospholipid syndrome, antiphospholipid antibodies

\section{Introduction}

Antiphospholipid syndrome (APS) is diagnosed when a patient meets at least one clinical and one laboratory criterion. The clinical criteria are (1) one or more clinical episodes of arterial, venous, or small vessel thrombosis in any tissue or organ confirmed by imaging or histopathology or (2) pregnancy morbidity. The laboratory criteria, all of which involve the presence of antiphospholipid (aPL) antibody, are (1) positive lupus anticoagulant (LA) antibody, (2) moderate to high levels of isotype IgG or IgM anticardiolipin (aCL) antibody, or (3) isotype IgG or IgM anti-beta-2 glycoprotein I (anti$\beta_{2}$ GPI) antibody on at least two occasions at least 12 weeks apart. ${ }^{1-7}$ In a review by Cervera, ${ }^{4}$ the estimated annual incidence of APS was 5 new cases per 100,000 people, and its prevalence increased with age. We herein present a case involving a very young patient with catastrophic APS (CAPS) who developed multiple thromboembolic events and positive LA antibodies.

ence: Jariya Waisayarat, Department of Pathology, Faculty of Medicine, Ramathibodi Hospital, Mahidol University, 270 Rama VI Road, Rajathevee, Bangkok 10400, Thailand

Tel $+662201 \quad 1432$

Fax +6623547266

Email jariya.wai@mahidol.ac.th

\section{Case report}

A 12-year-old Thai boy first presented to a local hospital with swelling and pain in both legs. Computed tomography angiography (CTA) revealed acute total occlusion 
of the right mid-femoral artery and left popliteal artery, pulmonary embolism (PE) in the left inferior pulmonary vein, and left renal vein thrombosis. He was treated by surgical embolectomy; however, his limb ischemia did not resolve, and he underwent bilateral limb amputation.

A laboratory investigation revealed LA antibody positivity. The protein $\mathrm{C}$ level, protein $\mathrm{S}$ level, antithrombin activity, homocysteine level, aCL antibody level, anti$\beta_{2}$ GPI antibody level, factor VIII activity, and lipoprotein (a) level were within the reference range. An autoimmune study revealed normal levels of anti-double-stranded DNA antibodies, anti-Sm antibodies, and complements. Therefore, CAPS was suspected. The patient was treated with plasmapheresis for 5 days, pulse methylprednisolone for 3 days, intravenous immunoglobulin at a dose of $2 \mathrm{~g} /$ $\mathrm{kg}$, and standard heparin. One month after the treatment, CTA showed a slightly decreased thrombus size in the renal vein and newly developed thrombosis in the right inferior pulmonary vein and right atrium. He then continued treatment with low-molecular-weight heparin and prednisolone. Three months later, he developed dyspnea and orthopnea. An echocardiogram revealed a $3.6-\times 3.4-\mathrm{cm}$ thrombus in the right atrium, and CTA showed bilateral renal artery stenosis. The thrombus was surgically removed. Pulse methylprednisolone, milrinone, dobutamine, and standard heparin were administered.

After the patient showed clinical improvement, the medications were changed to digoxin and enoxaparin. The patient then was referred to Ramathibodi Hospital for further treatment and diagnosis. At admission, physical examination revealed tachycardia and an oxygen saturation of $97 \%$. CTA showed a $2.7-\times 4.9-\times 4.0-\mathrm{cm}$ thrombus in the right atrium protruding into the right ventricle and acute $\mathrm{PE}$ in the subsegmental arteries. Pulse methylprednisolone, cyclophosphamide, intravenous immunoglobulin, and heparin were given. Further investigation of both perinuclear and cytoplasmic antineutrophil cytoplasmic antibodies was negative. Ten days after treatment, the patient's clinical symptoms improved. He continued treatment with enoxaparin, aspirin, prednisolone, and sildenafil for pulmonary hypertension. His anti-factor Xa level was within the therapeutic range at 0.9 to $1.0 \mathrm{IU} / \mathrm{dL}$. Four months later, after the fourth course of cyclophosphamide, he developed dyspnea and was found to have pericardial effusion caused by pulmonary hypertension. Pericardiocentesis was performed. CTA revealed a new thrombus in the pulmonary artery at the right lower segment and persistence of the thrombus in the right atrium. During hospitalization, he developed progressive dyspnea secondary to PE and pulmonary hypertension and subsequently died of heart failure.

Autopsy revealed a 22.5 -cm-long surgical wound in the chest wall from the sternotomy. Fibrinous pericarditis (Figure 1A) with about $30 \mathrm{~mL}$ of serosanguinous-type pericardial effusion was detected in the pericardial cavity. A 5.5- $\times 4.0-\times 4.0-\mathrm{cm}$ intracardiac thrombus was observed in the right atrial chamber; the thrombus was attached to the posterior leaflet of the tricuspid valve and extended into the right ventricular chamber (Figure 1B). Microscopic examination revealed an organized thrombus (Figure 1C) with endocardial fibrosis. A pulmonary thromboembolus was grossly demonstrated in the branch of the left pulmonary artery (Figure 2A). Microscopic examination revealed the recanalized phase of the thrombus with the organizing phase at the periphery (Figure 2B and C). Numerous recent thrombi were also observed in the capillaries and small arterioles of the bilateral lungs and kidneys. However, no evidence of vasculitis, infection, or malignancy was found in any organs.

\section{Discussion and conclusion}

The diagnosis of APS is based on the presence of vascular thrombosis or recurrent pregnancy loss and persistent positivity for aPL, LA, aCL, or anti- $\beta_{2}$ GPI antibodies on two or more occasions at least 12 weeks apart. APS without an associated condition is called primary APS. Vascular involvement in patients with APS presents with various features such as PE, lower extremity venous thrombosis, stroke, and intestinal infarction. ${ }^{4}$ In their 10-year study of a large cohort of patients with APS, Cervera et $\mathrm{al}^{8}$ found that the most common thrombotic events were stroke, transient ischemic attack, deep vein thrombosis, and PE. Intracardiac thrombus is an uncommon finding in patients with APS. ${ }^{9-15}$ CAPS, also known as Asherson's syndrome, is a rare and severe form of APS defined as a rapidly progressive thromboembolic disease involving three or more organs. The disease mainly affects small vessels supplying organs and presents within a short period of time (usually $<1$ week). ${ }^{3,8,16}$ Its prevalence is $<1 \%$ of all APS cases. ${ }^{4,16-18}$

Although the pathogenesis of APS is poorly understood, a "two-hit theory" has been proposed as the mechanism underlying APS. This theory suggests that aPL antibodies act through many possible routes to create the necessary conditions for thrombosis, acting as the "first hit": (1) aPL antibodies bind to $\beta_{2}$ GPI expressed on the surface of endothelial cells, inducing the cells to change to a procoagulant and proinflammatory phenotype. (2) aPL antibodies 


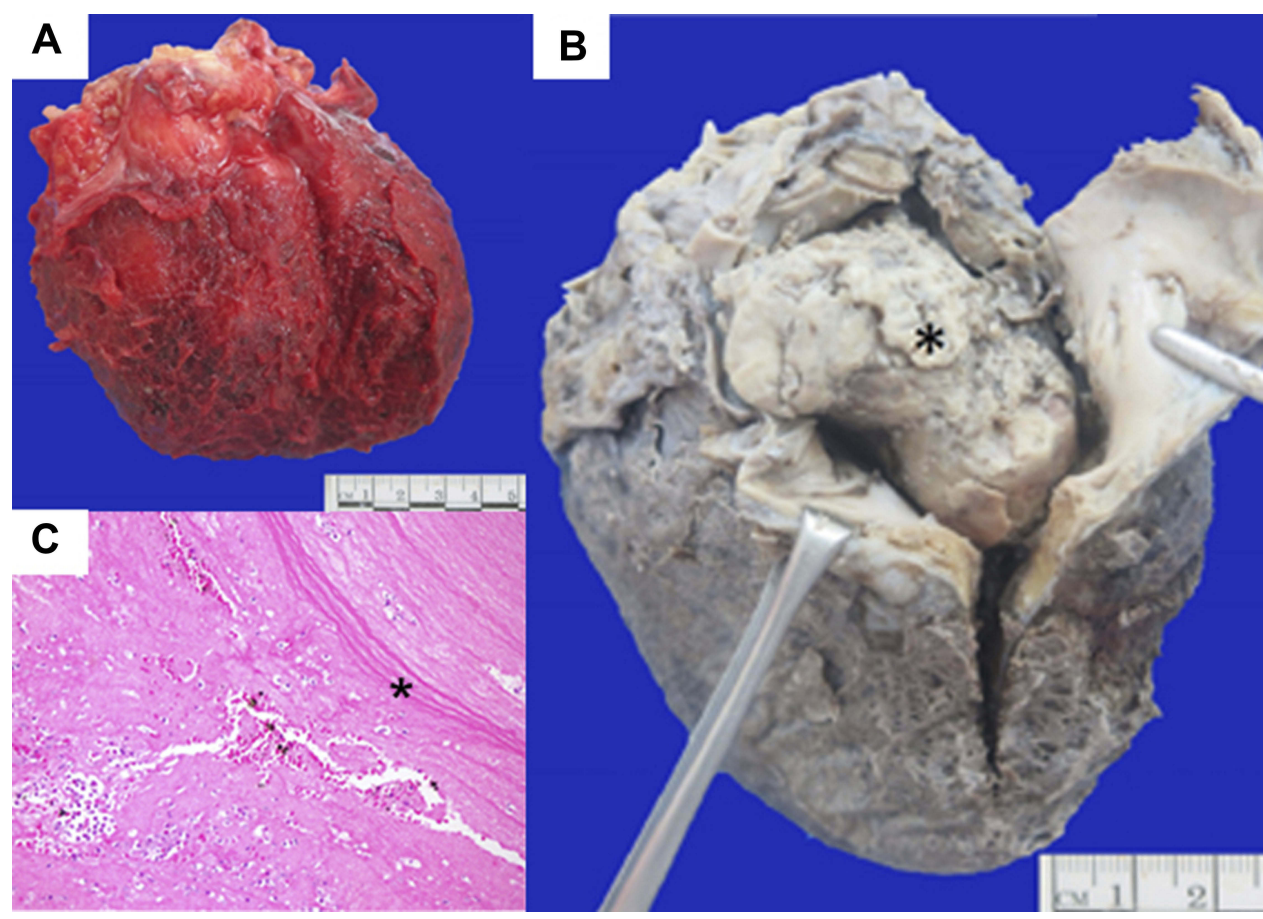

Figure I Heart with intracardiac thrombus. (A) Fresh specimen, anterior aspect. External appearance of the heart with fibrinous pericarditis. (B) Ten percent neutral formalin-fixed specimen, right lateral aspect. Opening of the right atrial chamber showed an intracardiac thrombus (asterisk) that extended into the right ventricular chamber. (C) Histopathological examination of the intracardiac thrombus showed a recanalized and organized (asterisk) thrombus. Hematoxylin and eosin; original magnification, $20 \times$.

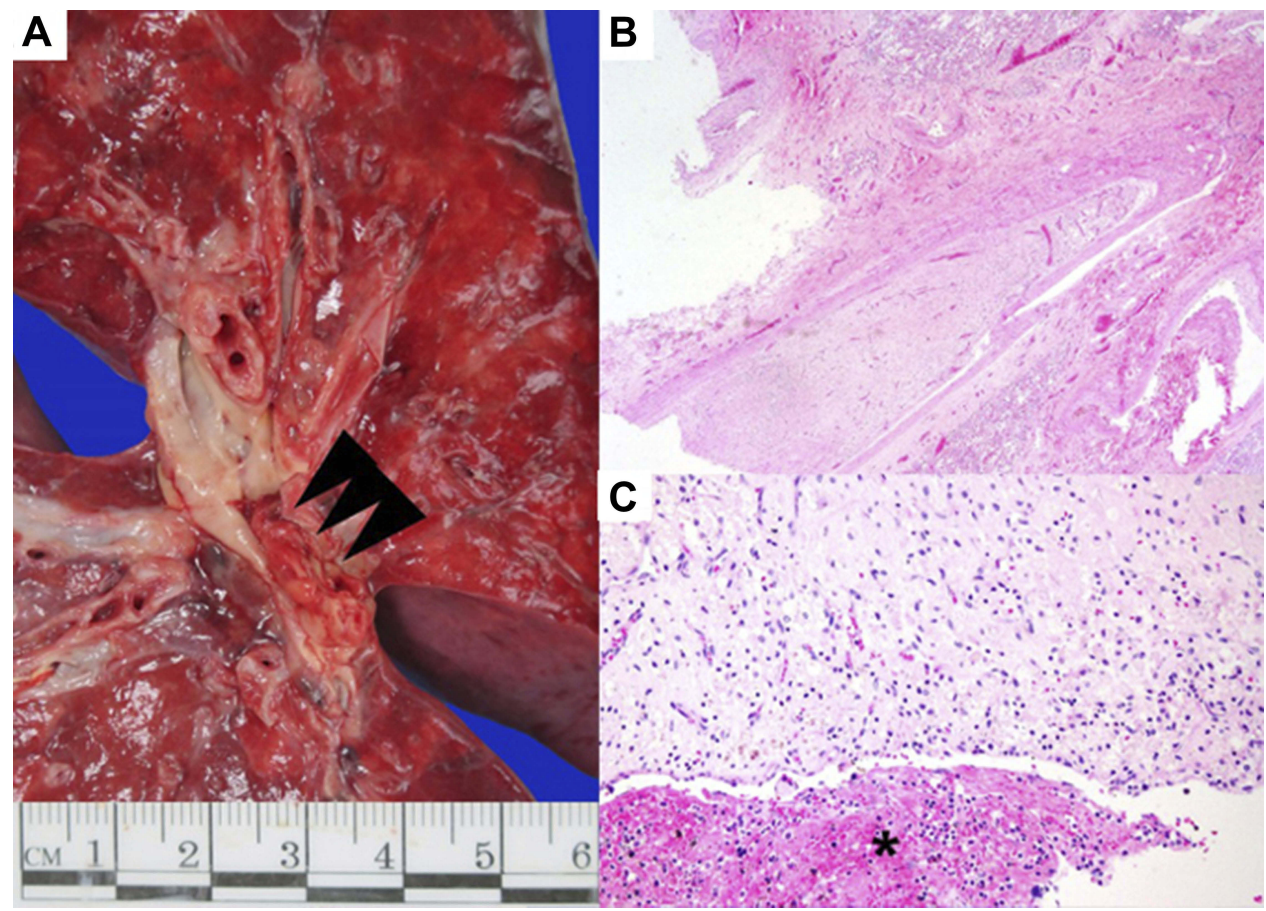

Figure 2 Left lung with pulmonary thromboembolus. (A) Fresh specimen. Red-white thrombus (arrowhead) in the descending branch of the left pulmonary artery. (B, C) Histopathological features of pulmonary thromboembolus showing organized (asterisk) and recanalized thrombus. Hematoxylin and eosin; original magnification, 20x in b and $100 \times$ in $\mathrm{c}$. 


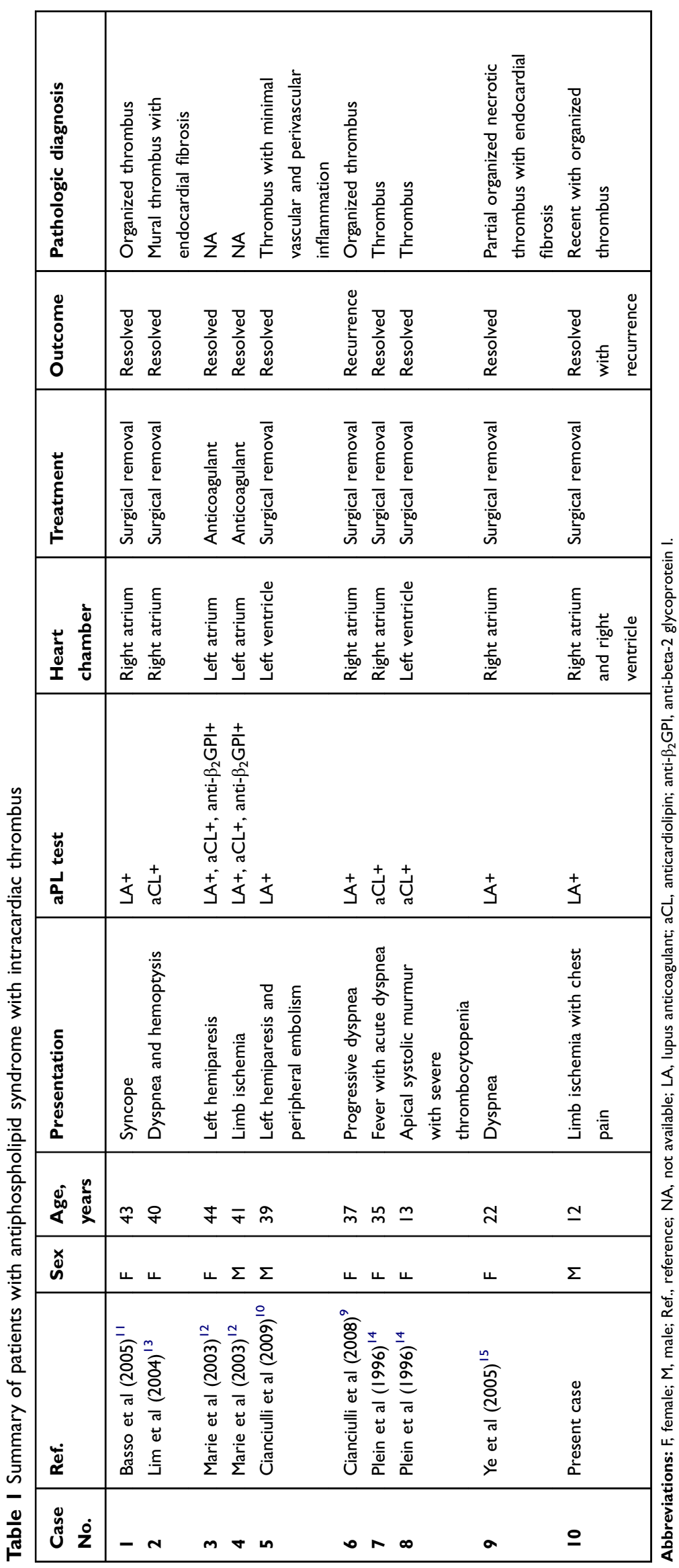


cause upregulation of platelet tissue factor expression by endothelial cells and monocytes, endothelial leukocyte adhesion, cytokine secretion, and prostaglandin E2 synthesis, all of which are processes that promote inflammation. (3) aPL antibodies bind to phospholipid-binding proteins on platelets, promoting platelet aggregation. (4) aPL antibodies inhibit fibrinolysis, binding of annexin A5 to anionic structures, and anticoagulant activity. These conditions alone are not sufficient to induce thrombosis, but a "second hit" that causes complement activation, such as that induced by infection, pregnancy, obesity, diabetes, or endothelial injury, is theorized to cause thrombus formation. ${ }^{19-21}$

Our patient had a history of vascular thrombosis (right femoral and left popliteal arteries, PE of the pulmonary vein, and left renal vein thrombosis) and a huge intracardiac thrombus in the right atrium extending into the right ventricle. An LA test was not performed 12 weeks later because of continued heparin treatment. Although he underwent surgical thrombus removal, he died of PE and heart failure. There was no evidence of a secondary cause of APS, such as systemic lupus erythematosus or autoimmune hemolytic anemia. Therefore, the patient was considered to have had primary APS classified as CAPS, with multiple vascular thromboses including intracardiac thrombus, a rare complication of APS. The exact mechanism underlying the development of intracardiac thrombus is not understood, but such thrombus formation can cause pulmonary and systemic embolic events. If the thrombus is small, it may be misinterpreted as a vegetation or intracardiac tumor such as an atrial myxoma by echocardiographic examination. Thus, the pathologist should be aware of these conditions as differential diagnoses when a surgical specimen from an intracardiac tumor is received. ${ }^{9,10}$ Although this condition is life-threatening, it is treatable. Management usually requires surgical thrombus removal. However, embolic events may recur; thus, intensive anticoagulation treatment and immunosuppressive therapies are required. The decision to perform surgical intracardiac thrombus removal in patients with APS must be made carefully because of the hemorrhagic risk factors accompanying APS and the risk of further thrombotic events and postoperative complications. ${ }^{22,23}$

One literature review summarized nine patients with APS with intracardiac thrombi (Table 1). Most of these patients were women aged 30 to 40 years. ${ }^{9-15}$ The most common location of the intracardiac thrombus was the right atrial chamber; in some cases, however, the thrombus was found in the left atrium and left ventricle. ${ }^{10,12,14}$ One of these patients was a female adolescent who had APS associated with underlying systemic lupus erythematosus. ${ }^{14}$ As mentioned above, intracardiac thrombi are treatable by surgical intervention or anticoagulation therapy. Most cases are resolved with a good clinical outcome, and recurrent events rarely occur. In the present case, however, PE recurred after resolution of the clinical symptoms, resulting in the patient's death. The postmortem thrombus was organized and showed no evidence of infection or malignant cells. The endocardium showed various degrees of endocardial fibrosis.

In conclusion, we have herein reported a case involving a 12-year-old Thai boy with CAPS diagnosed by thromboembolic events in several organs, including the extremities (history of bilateral lower limb amputation), lungs, renal vein, right ventricle, and right atrial chamber presenting with recurrent intracardiac thrombosis and pulmonary thromboembolism. The patient died of cardiac tamponade caused by clinically silent pericardial effusion followed by cardiogenic heart failure. Intracardiac thrombosis and pulmonary thromboembolism were confirmed by postmortem examination.

To our knowledge, the present report describes the youngest patient with APS with an intracardiac thrombus in both the right atrium and right ventricle. Despite the rarity of pediatric APS, physicians should keep in mind the possibility of this APS manifestation to ensure timely administration of effective treatments.

\section{Consent}

Written informed consent was obtained from the patient's parents for this case report and any accompanying images.

\section{Ethics approval}

This study was approved by the Institutional Review Boards of Mahidol University, in full compliance with International Guidelines for Human Research Protection such as Declaration of Helsinki, The Belmont Report, CIOMS Guidelines and the International Conference on Harmonization in Good Clinical Practice(MURA2019/27).

\section{Acknowledgments}

We wish to thank the patient's parents, who gave consent for us to study their beloved son for medical academic purposes. We acknowledge Associate Professor Pacharapan Surapolchai, MD, Department of Pediatrics, Faculty of Medicine, Thammasat University, for the clinical history of the patient. We are also grateful to our autopsy unit assistants for their help. We acknowledge Mr. Phu Waisayarat, a medical student of the Faculty of Medicine, King Mongkut's Institute of Technology Ladkrabang, for providing English 
proofreading services. Finally, we thank Angela Morben, DVM, ELS, from Edanz Group for editing a draft of this manuscript. The authors received no financial support for the research, authorship, or publication of this article.

\section{Author contributions}

All authors contributed toward data analysis, drafting and revising the paper, gave final approval of the version to be published and agree to be accountable for all aspects of the work.

\section{Disclosure}

The authors declare no conflicts of interest in this work.

\section{References}

1. Gómez-Puerta J, Cervera R. Diagnosis and classification of the antiphospholipid syndrome. J Autoimmun. 2014;48-49:20-25.

2. Levine J, Branch D, Rauch J. The antiphospholipid syndrome. $N$ Engl $J$ Med. 2002;346(10):752-763.

3. George D, Erkan D. Antiphospholipid syndrome. Prog Cardiovasc Dis. 2009;52(2):115-125.

4. Cervera R. Antiphospholipid syndrome. Thromb Res. 2017;151(Suppl 1):S43-S47.

5. Kaul M, Erkan D, Sammaritano L, Lockshin M. Assessment of the 2006 revised antiphospholipid syndrome classification criteria. Ann Rheum Dis. 2007;66(7):927-930.

6. Miyakis S, Lockshin M, Atsumi $\mathrm{T}$, et al. International consensus statement on an update of the classification criteria for definite antiphospholipid syndrome (APS). J Thromb Haemost. 2006;4(2):295306.

7. Moutsopoulos H, Vlachoyiannopoulos P. Antiphospholipid antibody syndrome. In: Longo D, Fauci A, Kasper D, Hauser S, Jameson J, Loscalzo J, editors. Harrison's Principles of International Medicine. 18th ed. New York: McGraw-Hill Education/Medical; 2011:2736-2738.

8. Cervera R, Serrano R, Pons-Estel G, et al. Morbidity and mortality in the antiphospholipid syndrome during a 10-year period: a multicentre prospective study of 1000 patients. Ann Rheum Dis. 2015;74(6):1011.
9. Cianciulli T, Saccheri M, Redruello H, et al. Right atrial thrombus mimicking myxoma with pulmonary embolism in a patient with systemic lupus erythematosus and secondary antiphospholipid syndrome. Tex Heart Inst J. 2008;35(4):454-457.

10. Cianciulli T, Saccheri M, Lax J, et al. Left ventricular thrombus mimicking primary cardiac tumor in a patient with primary antiphospholipid syndrome and recurrent systemic embolism. Cardiol $J$. 2009;16(6):560-563.

11. Basso C, Bottio T, Rubino M, et al. Antiphospholipid syndrome and right atrial mass. J Thorac Cardiovasc Surg. 2005;130(5):1462-1463.

12. Marie I, Derumeaux G, Delahaye F, et al. Intracardiac thrombi in primary antiphospholipid syndrome: two case reports. Eur J Intern Med. 2003;14(8):504-508.

13. Lim E, Wicks I, Roberts L. Intracardiac thrombosis complicating antiphospholipid antibody syndrome. Intern Med J. 2004;34(3):135-137.

14. Plein D, Van Camp G, Efira A, Brunet A, Vandenbossche J. Intracardiac thrombi associated with antiphospholipid antibodies. $J$ Am Soc Echocardiogr. 1996;9(6):891-893.

15. Ye Z, Yu C, Hsueh C, Leu H, Chen J, Lin S. Antiphospholipid syndrome presenting as intracardiac thrombus with pulmonary embolism. Circ J. 2005;69(10):1290-1292.

16. Cervera R, Bucciarelli S, Plasín M, et al. Catastrophic antiphospholipid syndrome (CAPS): descriptive analysis of a series of 280 patients from the "CAPS registry.". J Autoimmun. 2009;32(34):240-245.

17. Rodríguez-Pintó I, Moitinho M, Santacreu I, et al. Catastrophic antiphospholipid syndrome (CAPS): descriptive analysis of 500 patients from the International CAPS registry. Autoimmun Rev. 2016;15 (12):1120-1124.

18. Cervera R, Espinosa G, Bucciarelli S, Gómez-Puerta JA, Font J. Lessons from the catastrophic antiphospholipid syndrome (CAPS) registry. Autoimmun Rev. 2006;6(2):81-84.

19. Erkan D, Lockshin MD. New approaches for managing antiphospholipid syndrome. Nat Clin Pract Rheumatol. 2009;5(3):160-170.

20. Nalli C, Andreoli L, Casu C, Tincani A. Management of recurrent thrombosis in antiphospholipid syndrome. Curr Rheumatol Rep. 2014;16(3):405.

21. Meroni P, Borghi M, Raschi E, Tedesco F. Pathogenesis of antiphospholipid syndrome: understanding the antibodies. Nat Rev Rheumatol. 2011;7(6):330-339.

22. Egolum U, Stover D, Anthony R, Wasserman A, Lenihan D, Damp J. Intracardiac thrombus: diagnosis, complications and management. Am J Med Sci. 2013;345(5):391-395.

23. Ortel TL, Erkan D, Kitchens CS. How I treat catastrophic thrombotic syndromes. Blood. 2015;126(11):1285-1293.
Vascular Health and Risk Management

\section{Publish your work in this journal}

Vascular Health and Risk Management is an international, peerreviewed journal of therapeutics and risk management, focusing on concise rapid reporting of clinical studies on the processes involved in the maintenance of vascular health; the monitoring, prevention and treatment of vascular disease and its sequelae; and the involvement

\section{Dovepress}

of metabolic disorders, particularly diabetes. This journal is indexed on PubMed Central and MedLine. The manuscript management system is completely online and includes a very quick and fair peerreview system, which is all easy to use. Visit http://www.dovepress. com/testimonials.php to read real quotes from published authors. 